\title{
Commentary
}

\section{MANAGING ULCERATIVE COLITIS BY INCREASING HYDROGEN PRODUCTION VIA ORAL ADMINISTRATION OF ACARBOSE}

\author{
Jian-Hong Zhu ${ }^{\text {a, }}$, De-qing Zhang ${ }^{\text {a, }}$, Wei-chang Chen ${ }^{\text {a, * }}$ \\ Department of Digestion Internal Medicine, the First Affiliated Hospital ,Soochow University,Su \\ Zhou, 215006 , P.R. China. \\ * E-mail: chenweichang2012@163.com \\ ${ }^{\&}$ Co-First author, contributed equally to this paper
}

\begin{abstract}
The objective of the study was to investigate ulcerative colitis management through oral administration of acarbose. Acarbose has gained importance as a drug used widely to treat Diabetes Mellitus Type 2,as it acts on the small intestine by competitively inhibiting enzymes that delay the release of glucose from complex carbohydrates, thereby specifically reducing postprandial glucose excursion. The main side-effect of treatment with Acarbose, flatulence, occurs when undigested carbohydrates are fermented by colonic bacteria, resulting in considerable amounts of hydrogen. We found that the enteric benefits of Acarbose are partly due to be their ability to neutralise oxidative stress via increased production of $\mathrm{H}_{2}$ in the gastrointestinal tract. Therefore, some symptoms of ulcerative colitis in human beings can be ameliorated by Acarbose.
\end{abstract}

Keywords: Acarbose,hydrogen, Ulcerative colitis.

\section{Introduction}

Ulcerative Colitis (UC) is a chronic inflammatory disease affecting the rectum and the colon to a variable extent and exerts a substantially negative impact on the quality of life of affected patients. Some causes are still not known. Patients ailing from this disease have a $10 \%$ increased risk of developing colorectal carcinoma. Some progress has been made in the treatment of UC in the past few decades and many conventional drugs and therapies such as sulfasalazine, corticosteroids and immunosuppression are used to treat UC. Each therapy aims at the different etiological aspects of UC (Hawthorne et al., 2008; Carty et al., 2003). Unfortunately, all these medicines have side-effects. People suffer from these severe adverse effects and the quality of life goes down considerably. The course of UC is characterised by periods of remission interspersed with exacerbations (Solberg et al., 2008).

The expression of UC is generally believed to be an interplay of environmental, genetic and immunological factors (Fries et al., 2011). However, there is still not an integrated concept which explains the initiating event(s) and/or fundamental abnormalities in UC related to the pathophysiological changes. It does seem certain that UC is amplified and propagated by an uncontrolled and sustained host immune response, as the disease is paralleled by an extensive inflammatory infiltrate in the lamina propria, consisting of polymorphonuclear neutrophils, eosinophils and plasma cells. The effects from such excessive and enduring mucosal immune activation and tissue injury are still not fully confirmed. There are only a limited number of effects or mechanisms, including reactive oxygen metabolites (ROM), which may be responsible for the excessive cellular/tissue damage, chronic inflammation and destruction of normal tissue that is observed in UC (Karp and Koch, 2006). Impaired antioxidant activity is implicated as one of pathogenic causes of UC. Inflamed tissue generates hydroxyl radicals, most cytotoxic reactive oxygen species (ROS), which up-regulate TNF- $\alpha$ expression through NF-kB, signalling the 
http://dx.doi.org/10.4314/ajtcam.v10i5.23

pathway and the activation of NADPH-Oxygenase (NOX) expression, which in turn increases ROS production making this a vicious cycle (Gloire et al., 2006; Moe et al., 2006).

In principle, excessive generation of ROM may lead to the attack and damage of all cellular and extracellular components (Roessner et al., 2008). However, it is important to realise that the high reactivity and short half-life of ROM implies that the inflicted tissue damage is generally close to the site of ROM generation. In UC, activated macrophages and neutrophils produce excessive ROS aggregate in the inflamed gut and severe oxidative stress occurs subsequently. When the damage exceeds the antioxidative ability of the intestinal antioxidant defence system, ROS causes irreversible damage to membrane lipids, proteins, and DNA, making the oxidative stress injuries in patients with UC worse (Grisham and Granger, 1998).

Since both inflammation and antioxidant processes are antagonistic, antioxidants are viewed as a promising therapy for UC as they can significantly alleviate the symptoms of these processes, that is, body weight loss, visible faecal blood and diarrhoea (Oz et al., 2008).

\section{Molecular hydrogen (H2): a novel antioxidant and inflammation suppressor}

In recent years, experimental evidence has proven that molecular hydrogen $\left(\mathrm{H}_{2}\right)$ acts as a novel antioxidant and inflammation suppressor due to the following properties: (1) $\mathrm{H}_{2}$ permeates cell membranes and targets cellular organelles, including the mitochondria and nuclei; (2) $\mathrm{H}_{2}$ specifically targets detrimental ROS, such as hydroxyl (OH) and per-oxynitrite (ONOO-), while undergoing metabolic oxidation-reduction reactions with the less-potent ROS, such as $\mathrm{O}_{2}^{-}, \mathrm{H}_{2} \mathrm{O}_{2}$ and nitric oxide (NO) which have well defined physiological roles (Chen et al., 2011). Therefore, unlike the other antioxidant supplements with strong reductive reactivity, $\mathrm{H}_{2}$ is mild enough to work without disturbing metabolic oxidation-reduction reactions or disrupting Oxidative Stress (OS) important for cell signalling. Additionally, $\mathrm{H}_{2}$ is electrically neutral and much smaller than the oxygen molecule; so, that makes it easier to penetrate membranes and enter cells and organelles such as the nucleus and the mitochondria. This factor is particularly important, as the latter is the primary site of ROS generation and notoriously difficult to target, and hydrogen could diffuse freely within the mitochondria, without any side effects (Ohta, 2002). $\mathrm{H}_{2}$ can be administered orally in the form of $\mathrm{H}_{2}$-dissolved saline. Mikihito Kajiya reported that the administration of saturated hydrogen water for 7days reduced the levels of several biomarkers of proinflammatory cytokines, such as IL-12, TNF- $\alpha$ and IL-1 $\beta$ (Kajiya et al., 2009). In other experiments, hydrogen gas treatment has significant protective effects on schistosomiasis-associated chronic liver inflammation, and $\mathrm{H}_{2}$-rich saline treatment significantly attenuates the severity of L-Arg-induced acute pancreatitis by ameliorating the increased serum amylase activity, inhibiting neutrophilic infiltration and lipid oxidation (Chen et al., 2010). Therapeutic effects of $\mathrm{H}_{2}$ have been confirmed in the cell damage after stroke, ischemia-reperfusion injuries, transplantation injuries and other injuries related to oxidative stress (Xiao Chen et al., 2013; Ohsawa et al., 2007).

Thus, $\mathrm{H}_{2}$ can protect cells from oxidative stress injuries. In the inflammation process, $\mathrm{H}_{2}$ mediates suppression of proinflammatory cytokines, especially IL-1 $\beta$, TNF- $\alpha$ and IL-6 in inflammatory tissues. This research suggests that persistent intake of $\mathrm{H}_{2}$ has the potential to reduce oxidative stress and may actually ameliorate symptoms caused by Ulcerative Colitis.

\section{Acarbose facilitates $\mathrm{H}_{2}$ generation in gastrointestinal tract which may help ameliorate symptoms of Ulcerative Colitis}

Acarbose is an $\alpha$-glucosidase inhibitor acting specifically at the level of postprandial glucose excursion. This compound lowers $\mathrm{HbA}_{1 \mathrm{c}}$ by $0.5-1 \%$ in patients with Type 2 diabetes, either used as mootherapy in combination with other Oral Hypoglycemic drugs or Intra Muscular Insulin. In those with impaired glucose tolerance (IGT), it reduces the incidence of newly diagnosed diabetes by $36.4 \%$ (Gil et al., 2013). Furthermore, it has beneficial effects on obesity, reduction in blood pressure and triglycerides, and down-regulates biomarkers of low-grade inflammation (Hanefeld et al., 2004). The major 
http://dx.doi.org/10.4314/ajtcam.v10i5.23

side-effect of treatment with $\alpha$-glucosidase inhibitors, flatulence, occurs when undigested carbohydrates are fermented by colonic bacteria, resulting in gas formation. The reason for flatulence is thought to be the action in the small intestine which competitively inhibits enzymes, delaying the release of glucose from complex carbohydrates, thereby specifically reducing post-prandial glucose excursion. This delay leads to spontaneous production of $\mathrm{H} 2$ gas in the human body via the fermentation of undigested carbohydrates by the resident enterobacterial flora (Yoshihiko et al., 2009; Ladas et al., 1992)

$\mathrm{H}_{2}$ is not produced endogenously in mammalian cells, since the hydrogenase activity responsible for the formation of $\mathrm{H}_{2}$ gas is absent, thereby leaving the aforementioned fermentation as the method of production in the gut. $\mathrm{H}_{2}$ is transferred to the portal circulation and excreted through the breath in significant amounts (Michael and Levitt, 1969). Yoshihiko Suzuki reported that Acarbose treatment significantly increases the amount of exhaled $\mathrm{H}_{2}$ by testing eleven healthy volunteers (10 males and 1 female) who were administered Acarbose at a dosage of $300 \mathrm{mg} / \mathrm{day}$ ( $100 \mathrm{mg}$ three times a day) for 4 days under free-feeding conditions (Yoshihiko et al., 2009). Kajiyama treated patients with type 2 diabetes or impaired glucose tolerance with $900 \mathrm{ml} /$ day (300ml three times a day) $\mathrm{H}_{2}$-dissolved water. After drinking $300 \mathrm{ml} \mathrm{of} \mathrm{H}_{2}$-dissolved water, the exhaled $\mathrm{H}_{2}$ gas concentration reached a maximum of $56 \pm 27.8 \mathrm{ppm}$ at $15 \mathrm{~min}$, and returned to the baseline level at the 150 min mark (Kajiyama et al., 2008). In addition, $\mathrm{H}_{2}$ gas helped to reduce levels of oxidative stress biomarkers and improved glucose metabolism in patients with type 2 diabetes mellitus or impaired glucose tolerance. Considering the aforementioned evidence, gastrointestinal tract derived $\mathrm{H}_{2}$, which is closely related to reduced cardiovascular events, could reduce general oxidative stress injuries in other organ systems as well (Yoshihiko et al.,2009). We assume that the $\mathrm{H}_{2}$ gas released as a side effect Acarbose might be the key to the symptom alleviation in UC by reducing Myeloperoxidase (MPO) levels.

According to the published data in 2009, Ulcerative Colitis induced in mice by dextran sodium sulphate (DSS) was treated with saturated hydrogen water for 7 days. The study demonstrated that $\mathrm{H}_{2}$ can attenuate DSS-induced colitis by down-regulating the expression of proinflammatory cytokines, as well as suppressing the infiltration of macrophages in the colonic lesions (Kajiya et al., 2009). The administration of $\mathrm{H}_{2}$ remarkably reduced the clinical symptoms of DSS-induced colitis, that is, body weight loss, visible faecal blood, diarrhoea, colitis severity and shortening of colon length. Histopathological evaluation further supports the effects of $\mathrm{H}_{2}$ on the prevention of DSS-mediated destruction of epithelial crypt structure.

These observations clearly indicate that the amounts of $\mathrm{H}_{2}$ gas generated by Acarbose are sufficient to reduce systemic oxidative stress. Oral administration of Acarbose may be superior to drinking $\mathrm{H}_{2}$-rich water in terms of maintenance of the appropriate $\mathrm{H}_{2}$ gas levels in the body.

\section{Conclusion}

Based on these observations and experimental results, $\mathrm{H}_{2}$ has been proven effective in DSS-induced mice colitis and Acarbose does generate $\mathrm{H}_{2}$ in gastrointestinal tract in sufficient levels. Therefore, we hypothesise that Acarbose may be a novel and promising therapeutic option for Ulcerative Colitis as an indirect antioxidant. It may significantly restrict inflammation and alleviate clinical symptoms, improving the quality of life of chronic sufferers of Ulcerative Colitis. Although Acarbose probably has many other beneficial antioxidant effects on other diseases such as transplantation-induced organs injury, cardiovascular diseases, cerebrovascular accidents, among others, further research should be carried out to perfectly understand the working of this drug and its Mechanism of Action.

Conflict of interest: None declared.

\section{References}

1. A. B. Hawthorne, G Rubin, S.(2008).Ghosh.Review article: medication non-adherence in ulcerative colitis - strategies to improve adherence with mesalazine and other maintenance therapies.Alimentary Pharmacology \& Therapeutics.27:1157-1166. 

http://dx.doi.org/10.4314/ajtcam.v10i5.23

2. A Roessner, D Kuester, P Malfertheiner. 2008.Oxidative stress in ulcerative colitis-associated carcinogenesis. Research and Practice.204:511-524.

3. Carty E, Rampton DS. (2003). Evaluation of new therapies for inflammatory bowel disease. J Clin Pharmacol.56:351-61.

4. Chen H, Sun YP, Li Y, Liu WW, Xiang HG, Fan LY, Sun Q, Xu XY, Cai JM, Ruan CP, Su N, Yan RL, Sun XJ, Wang Q. 2010.Hydrogen-rich saline ameliorates the severity of L-arginine-induced acute pancreatitis in rats. Biochem Biophys Res Commun.393:308-313.

5. Chen X, Zuo Q, Hai Y, Sun XJ. 2011. Lactulose: An indirect antioxidant ameliorating inflammatory bowel disease by increasing hydrogen production. Medical hypotheses. 76:325-32.

6. Fries W, Comunale S. 2011.Ulcerative Colitis:Pathogenesis.Current Drug Targets. 12:1373-1382.

7. Gil V, Parsons S, Gallego D, Huizinga J, Jimenez M. (2013).Effects of hydrogen sulphide on motility patterns in the rat colon. British Journal of Pharmacology. DOI: 10.1111/bph.12100

8. Gloire G, Legrand-Poels S, Piette J. 2006.NF-kappaB activation by reactive oxygen species: fifteen years later. Biochem Pharmacol.72:1493-1505.

9. Grisham MB, Granger DN. 1998.Neutrophil-mediated mucosal injury: role of reactive oxygen metabolites. Dig Dis Sci.33:6S-15S.

10. Hanefeld M., Cagatay M., Petrowitsch T. (2004).Acarbose reduces the risk for myocardial infarction in type 2 diabetic patients: meta-analysis of seven long-term studies. European Heart Journal.25: 10-16.

11. .IC Solberg, I Lygren, J Jahnsen . (2008).Clinical course during the first 10 years of ulcerative colitis: results from a population-based inception cohort (IBSEN Study). Scandinavian Journal of Gastroenterology. 27:1157-1166.

12. Kajiya M, Silva MJ, Sato K, Ouhara K, Kawai T. 2009.Hydrogen mediates suppression of colon inflammation induced by dextran sodium sulfate. Biochem Biophys Res Commun 386:11-15.

13. Kajiyama S, Hasegawa G, Asano M, Hosoda H, Fukui M, Nakamura N, Kitawaki J, Imai S, Nakano K, Ohta M, Adachi T, Obayashi H, Yoshikawa T. (2008).Supplementation of hydrogen-rich water improves lipid and glucose metabolism in patients with type 2 diabetes or impaired glucose tolerance. Nutr. Res.28:137-143.

14. Karp SM, Koch TR. 2006.Oxidative stress and antioxidants in inflammatory bowel disease. Dis Mon .52:199-207.

15. Michael D, Levitt, M D. (1969).Production and excretion of hydrogen gas in man. New England Journal of Medicine.281:122-127.

16. Moe KT, Aulia S, Jiang F, Chua YL, Koh TH, Wong MC, Dusting GJ. 2006.Differential upregulation of Nox homologues of NADPH oxidase by tumor necrosis factoralpha in human aortic smooth muscle and embryonic kidney cells. J Cell Mol Med.10:231-239.

17. Ohsawa I, Ishikawa M, Takahashi K, Watanabe M, Nishimaki K, Yamagata K, Katsura K, Katayama Y, Asoh S, Ohta S.(2007).Hydrogen acts as a therapeutic antioxidant by selectively reducing cytotoxic oxygen radicals.Nature Medicine.13:688 - 694.

18. Oz HS, Chen TS, McClain CJ, de Villiers WJ. 2005.Antioxidants as novel therapy in a murine model of colitis. J Nutr Biochem.16:297-304.

19. S D Ladas, A Frydas, A Papadopoulos, SA Raptis. (1992). Effects of $\alpha$-glucosidase inhibitors on mouth to caecum transit time in humans. Gut.33:1246-1248.

20. S Ohta. 2012.Molecular hydrogen is a novel antioxidant to efficiently reduce oxidative stress with potential for the improvement of mitochondrial diseases. Biochimica et Biophysica Acta.doi:10.1016/j.physletb.2003.10.071

21. Suzuki Y, Sano M, Hayashida K, Ohsawa I, Ohta S, Fukuda K.(2009). Are the effects of a-glucosidase inhibitors on cardiovascular events related to elevated levels of hydrogen gas in the gastrointestinal tract? FEBS Letters.583:2157-2159.

22. Xiao Chen, Xiao Zhai, Jiazi Shi, Wen Wu Liu, Hengyi Tao, Xuejun Sun, Zhimin Kang.(2013). Lactulose Mediates Suppression of Dextran Sodium Sulfate-Induced Colon Inflammation by Increasing Hydrogen Production. Digestive Diseases and Sciences.DOI:10.1007/s10620-013-2563-7. 